\title{
Voiding urosonography with ultrasound contrast agents for the diagnosis of vesicoureteric reflux in children
}

\section{Procedure}

\author{
Kassa Darge
}

Received: 20 February 2007 /Revised: 23 April 2007 / Accepted: 1 May 2007 / Published online: 6 July 2007

(C) Springer-Verlag 2007

\begin{abstract}
Voiding urosonography (VUS) encompasses examination of the urinary tract with intravesical administration of US contrast agent (UCA) for diagnosis of vesicoureteric reflux (VUR). The real breakthrough for US examination of VUR came with the availability of stabilized UCAs in the mid-1990s. This article presents a comprehensive review of various procedural aspects of VUS. Different US modalities are available for detecting the echogenic microbubbles: fundamental mode, colour Doppler US, harmonic imaging and dedicated contrast imaging with multiple display options. The reflux is graded ( 1 to 5 ) in a similar manner to the system used in voiding cystourethrography (VCUG). The most commonly used UCA for VUS, Levovist, is galactose-based and contains air-filled microbubbles. The recommended concentration is $300 \mathrm{mg} / \mathrm{ml}$ at a dose of $5-10 \%$, or less than $5 \%$, of the bladder filling volume when using fundamental or harmonic imaging modes, respectively. There are preliminary reports of VUS using a second-generation UCA, SonoVue. Here the UCA volume is less than $1 \%$ of the bladder filling volume. There is no specific contraindication to intravesical administration of UCA. The safety profile of intravesical Levovist is very high with no reports of side effects over a decade of use in VUS.
\end{abstract}

Keywords Voiding urosonography.

Ultrasound contrast agent . Vesicoureteric reflux

\footnotetext{
K. Darge $(\bowtie)$

Department of Pediatric Radiology,

University Hospital Wuerzburg,

Josef-Schneider Str. 2/D31,

97080 Wuerzburg, Germany

e-mail: darge@roentgen.uni-wuerzburg.de
}

\section{Introduction}

Diagnostic imaging for vesicoureteric reflux (VUR) is a common procedure in children. Currently, three modalities are available for reflux diagnosis, namely voiding cystourethrography (VCUG), radionuclide cystography (RNC) and voiding urosonography (VUS). The last of these is carried out using US in combination with intravesical administration of US contrast agent (UCA). This allows the use of ionizing radiation to be avoided, which is not the case in RNC and VCUG. However, in VCUG a marked reduction in radiation dose has been achieved with the introduction of digital pulsed fluoroscopy.

The first attempts at the implementation of US for the diagnosis of VUR began in the mid-1970s. A comprehensive account of the evolution of this undertaking over the subsequent two decades has been presented by Darge [1]. The indirect methods for reflux diagnosis were based on US of the urinary tract, without administration of any kind of substance into the bladder. These included depicting various sonomorphological changes of the urinary tract as a result of VUR, detecting newly appearing or an increase in existing ureteral or pelvicalyceal dilatation during voiding and assessing ureteric jet changes with duplex and colour Doppler US. The direct means used to diagnose VUR required instilling different substances intravesically. The most frequently administered fluid was physiological saline solution. Ballooning of the renal pelvis during the filling of the bladder was the criterion for diagnosis of VUR. Application of air bubbles, by shaking the normal saline before administration or adding carbon dioxide, were also tried. US studies were also carried out, in which the empty bladder was solely filled with air. In addition to low diagnostic accuracy, all the above methods had major 
procedural drawbacks making them impractical for widespread integration into routine imaging.

The intravesical use of a UCA consisting of sonicated albumin (Albunex; Molecular Biosystems, San Diego, Calif.) for VUS in a child was first reported in 1994 [2]. Another UCA used in the past was Echovist (Schering, Berlin, Germany), which is composed of galactose with incorporated microbubbles [3]. Its very short imaging window of approximately $5 \mathrm{~min}$, however, prevented its routine application. The breakthrough in US diagnosis of VUR in children came about the mid-1990s with the availability of UCAs containing stabilized microbubbles. Levovist (Levograf, Schering Spain, Madrid, Spain; SHU508-A, Schering, Berlin, Germany) was the first such UCA to become available for clinical use in Europe. This opened the door for rapid development of VUS and its introduction as part of the routine diagnostic imaging option of VUR.

A number of different names and acronyms have been put forward to denote US examination for the diagnosis of VUR using intravesical UCAs. These include simply "sonography/ultrasound" [4-6], "reflux sonography" [7], "cystography" [8-10], "cystosonography" [11-15], "cystourethrosonography" [16] and "urosonography" [1723]. They are more often used in combination with one or more of the following terms: "echo-enhanced", "contrastenhanced" and "voiding". Depending on the US technique employed further descriptions such as "colour Doppler US" or "harmonic imaging" are added. The most widely applied name "voiding urosonography" with the abbreviation "VUS" was proposed for the first time in 2000 [24]. The selection of this particular designation was based on careful consideration of various factors. The prefix "uro-" is used to denote the bladder, ureters and kidneys. "Sonography" was chosen rather than "ultrasonography" as it is a shorter form. Thus "urosonography" correctly describes the fact that in this examination US of the bladder, ureters and kidneys takes place independent of the absence or presence of VUR. "Voiding" was selected instead of "micturating" as it is used more commonly in the medical literature. Despite the fact that for VCUG x-ray contrast agent is necessary, this is not directly added to the name VCUG in the form of, for example, contrast-VCUG. Likewise, the terms "contrast" or "echo-enhanced" were not added by default to the basic term "voiding urosonography". The abbreviation "VUS" is not only similar to the most common acronym used for the radiological reflux examination, namely VCUG, but had also not been used in the medical literature to denote something else [25]. For the sake of minimizing the confusion of names and facilitating communication, literature search and procedural standardization, the use of one name for the same procedure, "voiding urosonography" ("VUS") is recommended.
This article is part I of a comprehensive review of all currently available literature on VUS and prepares the ground for objective evaluation and decision-making. In this part (part I) a detailed procedural description is presented, including discussion of the pros and cons of the various examination steps and imaging modalities for VUS. The measures undertaken to optimize VUS are elaborated. Furthermore, studies dealing with adverse events of intravesical administration of UCAs are reviewed. In part II a review of comparative studies between VUS and $\mathrm{RNC} / \mathrm{VCUG}$ is presented [26].

\section{US contrast agent}

The most widely used UCA for VUS is Levovist. This firstgeneration UCA was introduced for intravenous (IV) use in the mid-1990s and from 1999 started to obtain approval for intravesical application in children successively in 13 European countries and Australia. Levovist consists of dry granules made of galactose and palmitic acid [27]. The granules easily disintegrate into microparticles upon preparation. They form porous structures, which are necessary for the formation of bubbles. Gas bubbles are formed within the pores during suspension of the granules, while dissolution of the galactose particles begins simultaneously. The Levovist bubble is a microbubble of air $-65 \%$ nitrogen and $35 \%$ oxygenstabilized by palmitic acid. One gram of Levovist granules contains $999 \mathrm{mg}$ D-galactose and $1 \mathrm{mg}$ palmitic acid. The concentrations of microbubbles in freshly prepared batches $(300 \mathrm{mg} / \mathrm{ml})$ are within the range of approximately $1-2 \times 10^{8}$ microbubbles per millilitre of suspension. The Levovist suspension should always be freshly prepared prior to intravesical administration. The steps of preparation, as outlined by the manufacturer, must be strictly adhered to in order to avoid any reduction of contrast enhancement due to improper handling [28]. The administration of freshly prepared UCA has to be carried out within $30 \mathrm{~min}$ [29].

\section{Concentration}

The recommended concentration of Levovist for VUS is $300 \mathrm{mg} / \mathrm{ml}$ [28]. This concentration has been used for VUS in most studies. A concentration of $200 \mathrm{mg} / \mathrm{ml}$ seems to result in faster dissipation of microbubbles, as found in invitro experiments (unpublished data). In only three studies has a concentration of $200 \mathrm{mg} / \mathrm{ml}$ been used [13, 15, 30]. Bosio initially used this concentration, but later changed to the higher one as the conspicuity of refluxing microbubbles in fundamental mode was inadequate [13]. In another study in adults with transplanted kidneys this concentration in combination with an increased volume, i.e. $10-15 \%$ of bladder filling, was used [30]. The combination of colour 
Doppler US with the burst technique administering $200 \mathrm{mg} / \mathrm{ml}$ selectively in infants has been reported, but the results using just this concentration were not analyzed separately [15]. The utilization of the higher concentration of $400 \mathrm{mg} / \mathrm{ml}$ has only been reported once without any additional advantages being mentioned [31]. Probably, the disadvantage would be an increase in UCA volume leading to an unwarranted increase in cost.

\section{Dosage}

The volume of UCA administered has been rather variable. Some have applied a fixed dose for all, independent of the patient's age, weight and bladder volume [4, 5]. In one study the volume was calculated in terms of body weight [13]. In most studies the volume of bladder filling is taken as the decisive factor $[1,12,28]$. This is very logical when considering intravesical administration of UCA and contrast enhancement of bladder content. It is possible to directly measure the bladder volume using the formula for an ellipse (length $\times$ width $\times$ height $\times 0.5$ ], note the total volume of normal saline administered in the bladder and also calculate in a simple way the maximum bladder capacity: volume in millilitres $=$ (age in years +2$) \times 30$ [32]. Furthermore, using the bladder volume for calculation of UCA dose makes comparison between studies much easier.

Nakamura et al. [21] carried out simultaneously VCUG and VUS in 56 children. The fundamental (i.e. conventional) imaging modality was used for VUS. They initially administered Levovist into an almost empty bladder followed by continuous infusion of the radiographic contrast agent. They simultaneously monitored the appearance of reflux during VUS, relating it to the concentration of the UCA in the bladder. The concentration of UCA in the bladder at one point in time ranged form $1.8 \%$ to $23 \%$. All false-negative results in the VUS were associated with a Levovist volume of $<5 \%$ of the total bladder filling. Thus a volume of UCA between $5 \%$ and $10 \%$ of the bladder filling is required when performing VUS using fundamental imaging. As there is a clear correlation between the experience of the sonographer and the sensitivity of VUS, it is advisable for beginners to start with the highest recommended volume of $10 \%$ and with increasing experience to reduce the amount, but without going below $5 \%$ of the bladder filling when using fundamental imaging. It seems possible to reduce the UCA volume when adding colour Doppler US [15]. There is clear evidence that when using harmonic imaging, 5\% or less of UCA with respect to the bladder filling will suffice [18, 33]. Preliminary experience with dedicated contrast imaging modalities such as cadence agent detection imaging (ADI) (Acuson; Siemens, Mountain View, Calif.) point to the potential of further dose reduction [34].
Physicochemical properties

UCAs were primarily developed for IV use and, consequently, preclinical studies were aimed at elucidating problems that could potentially have been encountered during administration via this route. Intravesical use is different and has its own peculiarities one must be aware of. An in-vitro study was carried out with Levovist to measure how variations in US machine setting, transducer choice, mode of application and bladder content may affect the microbubbles [35]. The effects of power output, transducer frequency and injection rate were found to be comparable to those during IV administration. The physicochemical properties of particular relevance for intravesical use are described below.

\section{Interaction with normal saline solution}

Unlike in VCUG and direct RNC, normal saline plays an important role in VUS. It is the actual bladder filling medium in which the microbubbles are injected. In an invitro study the median contrast duration when the UCA was mixed with normal saline solution from glass containers was $30 \mathrm{~s}$, whereas when the same UCA was added to normal saline solution from a plastic container the median contrast duration increased to $11 \mathrm{~min}$ [36]. None of the plastic containers were sealed under vacuum. In contrast, most of the glass containers were sealed under vacuum. The mean $\mathrm{pO}_{2}$ of the normal saline solutions from vacuumsealed containers was found to be $50 \%$ less than the $\mathrm{pO}_{2}$ of the normal saline solutions from plastic containers. The airfilled microbubbles of Levovist are permeable to air, and gas is exchanged with the atmosphere until equilibrium is reached [27]. Thus, in normal saline solution with low air saturation the microbubbles tend to collapse promptly [37]. Collapse of the microbubbles means that echo enhancers are not available. In practice, the use of normal saline solution from plastic containers is preferred.

\section{Interaction with urine}

Contrast duration of the UCA has been found to be more than four times longer in urine than in normal saline. It has been shown that simply adding urea to buffer the solution significantly increases contrast duration [38]. It is postulated that urea affects the bonding between water molecules and facilitates the formation of more microbubbles. Thus, having some urine in the bladder when performing VUS actually has a positive effect on imaging.

\section{Ascent of microbubbles in the ureter}

Once reflux of microbubbles takes place from the bladder into the distal ureter, the question as to whether the microbubbles 
can ascend passively to the proximal ureter and renal pelvis while the refluxed fluid stays in the distal ureter has been raised [39]. Given constancy of gravity, the density difference between gas and fluid and the viscosity of fluid, the velocity of ascent is proportional to the square of the microbubble radius. The typical microbubble radius of Levovist is $1-2.5 \mu \mathrm{m}$. Calculation of velocity of ascent indicates that for all practical purposes passive ascent can be excluded. Furthermore, in an in-vitro simulation of VUS with a set-up imitating the worst-case scenario $\left(90^{\circ}\right.$, glass tube (no adsorption), constant stirring of fluid suspension, and absence of counter-flow) the possibility of passive ascent was evaluated using an ultraviolet spectrometer for the detection of microbubbles. The result of this experiment was also negative. These findings suggest that the microbubbles in the ureter do not ascend passively and that reflux pressure is necessary for propagation, particularly in vivo with a constant counter-flow of urine from the renal pelvis to the bladder.

\section{Procedural details}

VUS entails four major basic steps: (1) scan of the urinary tract before contrast agent administration, (2) intravesical administration of prewarmed physiological saline solution and UCA, (3) scan of the urinary tract after administration of UCA, and (4) scan of the urinary tract after administration of UCA during and after voiding [28, 40]. Optionally, a transperineal US of the urethra and/or cyclical filling of the bladder may be added. Various US modalities are available for performing VUS. The main differences between the modalities are in the degree of conspicuity of the microbubbles and sensitivity of reflux detection.

US scan modalities

\section{Fundamental imaging}

This conventional modality in B-mode is the earliest and most widespread modality employed for VUS. In the case of fundamental imaging it is important to use the same scan planes and magnification of the ureters and renal pelves before and after UCA administration in order to facilitate comparison of the images (Fig. 1). This is particularly important when the reflux is not obvious.

\section{Colour Doppler US}

The microbubbles increase the backscattered signal from the urine giving it a characteristic chromatic effect, e.g. a set of blue and red punctiform colour collection [32]. This makes recognition of the urine flow direction easier and enhances the visualization of the microbubbles in the pelvicalyceal system. It is recommended that the Doppler US settings be optimized to perform VUS [31, 41]. Currently, there are five reports in which colour Doppler US alone or in combination with fundamental imaging have been used for VUS [11, 14, 31, 41, 42]. The diagnostic accuracy of colour Doppler US was compared with fundamental imaging in only one study [41]. In the same group of patients VUS without and with colour Doppler US followed by VCUG was carried out. With VCUG as the reference method, the diagnostic accuracy was found to be $96 \%$ with colour Doppler US and 90\% without. In another study, no significant increase was found in the sensitivity and specificity of VUS when adding colour Doppler US [42]. In one study a modified colour Doppler US examination was used employing a high mechanical index (MI) burst contrast technique based on stimulated acoustic emission (SAE), in which the microbubbles are made to burst creating strong acoustic signals [15]. It was possible to detect more reflux with the burst colour Doppler US compared to regular colour Doppler US or fundamental imaging and even VCUG. No systematic evaluation is available regarding the application of power Doppler US for VUS. Overall, adding optimized colour Doppler US to fundamental imaging seems to have some advantage and should be tried when other contrast-specific modalities are not available.

\section{Harmonic imaging}

The nonlinear propagation property of US waves is the basis of harmonic imaging [43]. This may be optimized to receive harmonics from either tissue or microbubbles, thus creating "tissue-specific harmonic imaging" or "contrastspecific harmonic imaging", respectively. Some US machines only use the second harmonic for imaging (narrow band), whereas others are capable of implementing a wider range of harmonics (wide band). There may be some degree of difference in image quality between these different technical approaches, in general the latter being of higher quality.

Harmonic imaging increases contrast and spatial resolution and also results in a reduction of artefacts [43]. The resulting images are clearer and crisper. The advantage of this modality compared to fundamental imaging for scanning the urinary tract in children has already been shown [44]. Due to attenuation, at a certain depth the positive effect of harmonic imaging will disappear. Regarding contrast-enhanced imaging, there is distinctly increased conspicuity of the microbubbles with harmonic imaging (Fig. 2). Air-filled bowel can be a disturbing factor, particularly when imaging the retrovesical space, requiring adjustment of the gain. Darge et al. [17] compared 
Fig. 1 Scans in fundamental mode before (a, c) and after (b, d) contrast agent administration of a dilated left distal ureter (a, b arrow) and pelvicalyceal system (c, d). In the postcontrast scans echogenic microbubbles fill the distal ureter (b) and are also detected in the pelvicalyceal system (d)
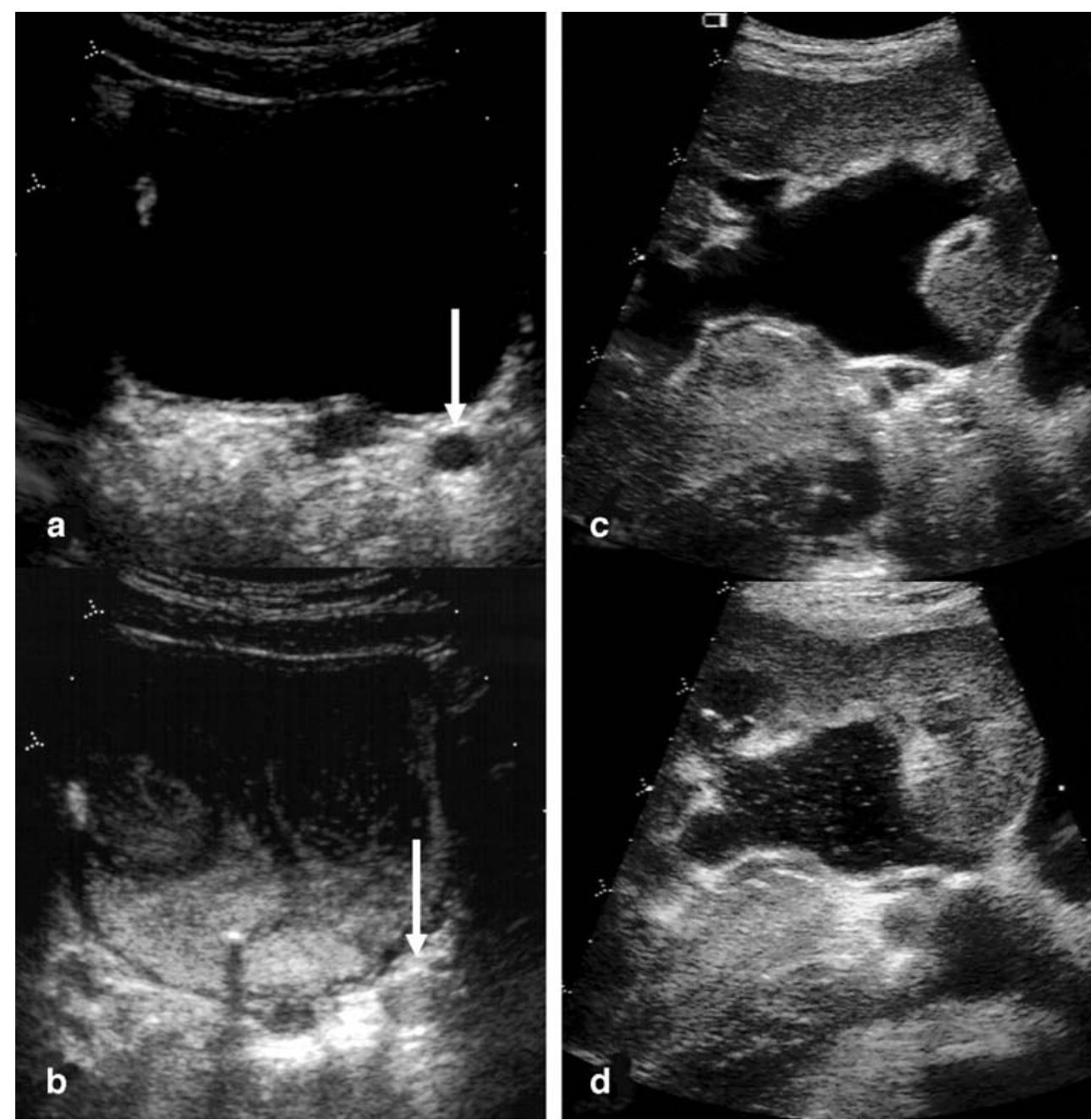

fundamental and harmonic imaging options in 54 children undergoing VUS. In all cases the conspicuity of the microbubbles was much higher with harmonic imaging than with the fundamental modality. Moreover, there was an increase in the reflux detection rate of $30 \%$ (from 19 to 27 pelvic-ureter units, PUUs). In this study the volume of Levovist used was $10 \%$ with respect to bladder filling. Even reducing the volume of UCA, more reflux episodes were detected in VUS with harmonic imaging than in VCUG [18, 33]. If available, harmonic imaging should be chosen in preference to the fundamental modality for VUS $[17,18,33]$.

\section{Dedicated high-MI contrast imaging with multiple display options}

In recent years, there have been major developments in US technologies for contrast-enhanced US. One such US modality uses high-MI imaging resulting in destruction of microbubbles and depicts the bubble destruction signature as a colour overlay with the possibility of visualizing the grey-scale image alone, the grey-scale image together with the microbubbles with colour overlay or just the microbubbles with colour overlay alone. Additional features may be real-time dual imaging in which two of the above modalities can be used in parallel and the possibility to switch between the three different presentations after having acquired an image in just one option (Figs. 3 and 4). This modality is named differently by different manufacturers: for example, agent detection imaging (ADI; Sequoia, Acuson Siemens), contrast tissue enhancement imaging (CTEI; Technos MPX, Esaote), rate subtraction imaging (RSI; Aplio, Toshiba), true agent detection (TAD; Logiq 9, GE), etc. [45]. The increase in microbubble conspicuity with this modality is so striking that even a beginner should find the examination easy to perform. The potential for further reduction of the dose of UCA and duration of examination are enormous and require future evaluation. If available, this US technique is preferred for VUS using Levovist. This feature is also available with low-MI imaging, which is important when using newer generation UCAs. 
Fig. 2 Scans after contrast agent administration in fundamental mode $(\mathbf{a}, \mathbf{c})$ and with harmonic imaging $(\mathbf{b}, \mathbf{d})$ of the bladder and right dilated ureter (a, b dotted circle) and a duplex kidney (c, d) with a multicystic dysplastic upper moiety. Reflux in the right ureter and in the lower moiety of the duplex kidney (grade II, arrow) are much more conspicuous with harmonic imaging $(\mathbf{b}, \mathbf{d})$. Note also the crisper depiction of the cysts in the upper moiety with harmonic imaging
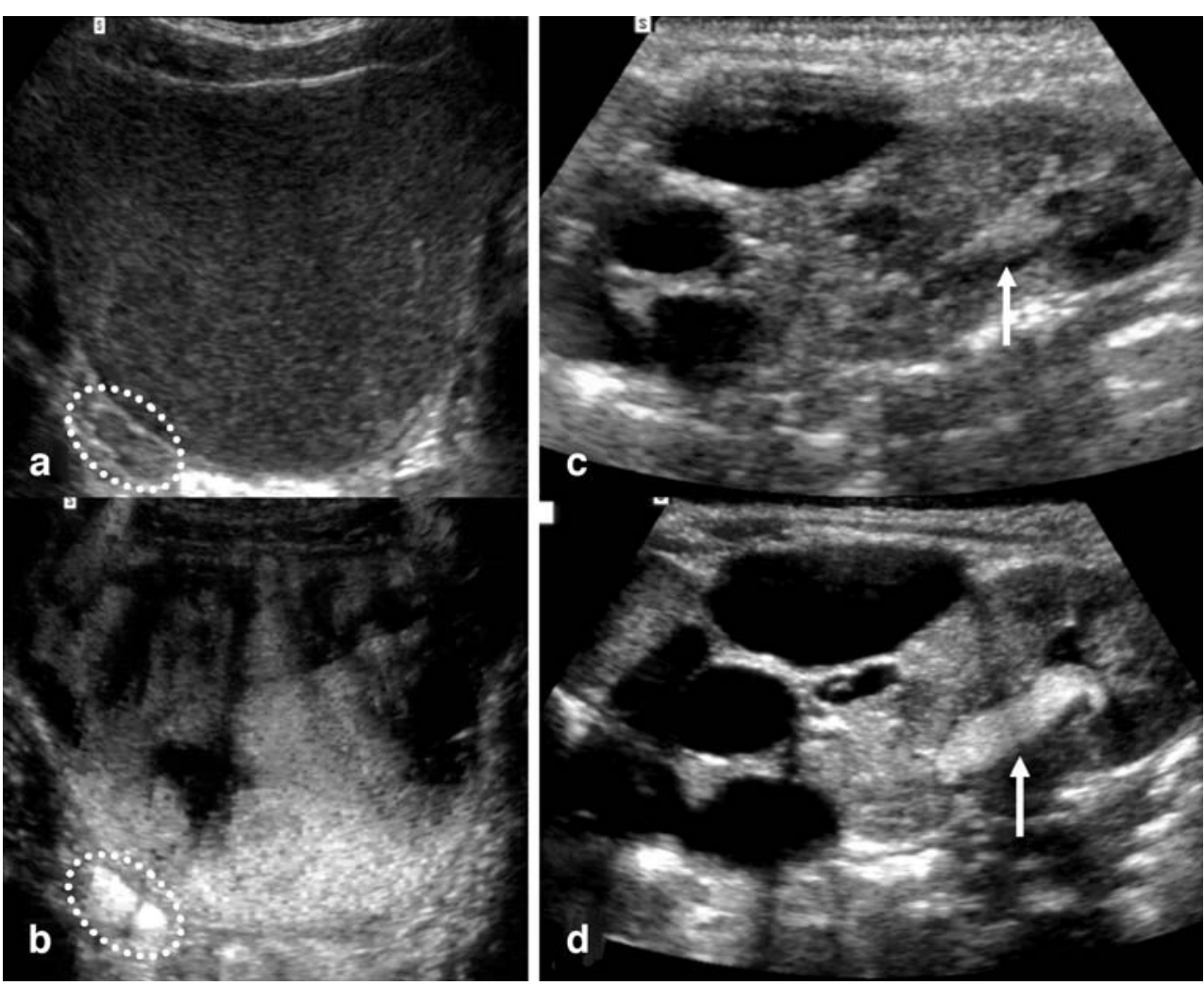

Procedural steps

\section{Precontrast scans of the bladder, ureters and kidneys}

The necessity to perform a detailed scan of the urinary tract arises when the VUS is combined with a follow-up US of the urinary tract, for example after pyelonephritis or when there is a need for comparison with the postcontrast images.
The scan before contrast agent administration should be carried out in accordance with standard US of the urinary tract in the supine position (and/or the prone position), in both the transverse and longitudinal planes [28]. Particular attention should to be paid to documentation of subtle changes in the retrovesical region, the vesicoureteric junction and any dilated ureter. The renal pelves (and/or the calyces) are imaged with maximum magnification. A

Fig. 3 VUS with the application of dedicated contrast imaging modality using high-MI (agent detection imaging, ADI). Reflux into the right ureter (arrow) and right renal pelvis (grade II). a Using grey-scale display alone the refluxing microbubbles are not easily demonstrated but the renal parenchyma is seen well. b Using "grey scale + contrast" visualization of both the reflux with a colour overlay and the bladder and kidney are noticeably improved. c Using "contrast only" the tissue part has been subtracted and only the microbubbles are seen

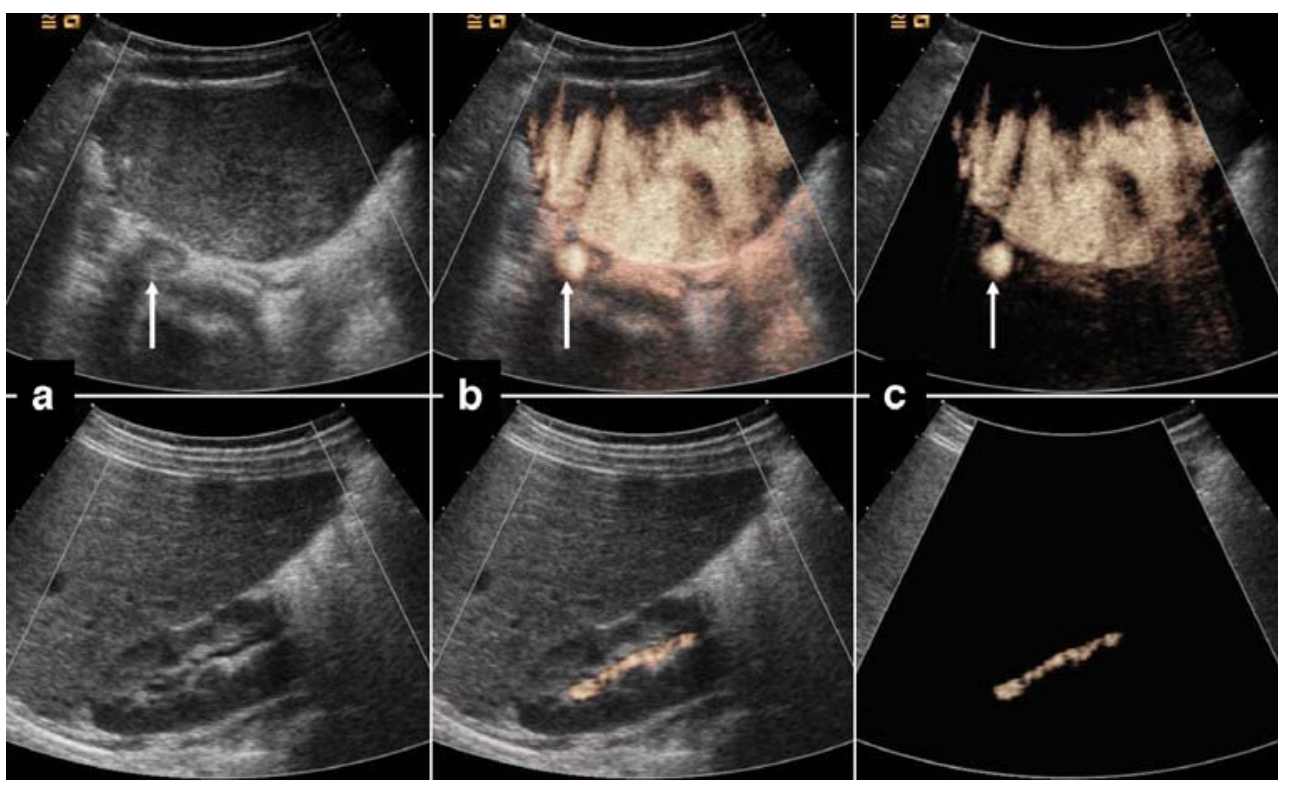


Fig. 4 VUS with the use of a dedicated contrast imaging modality using high-MI (agent detection imaging, ADI). Note the time and number of the images (arrow). Once just one image has been documented it is possible as a postprocessing option to switch between the different modalities for display: a "greyscale + contrast" and $\mathbf{b}$ "contrast only" options. In this case of grade III reflux marked intrarenal reflux is present in the upper pole (arrowhead)
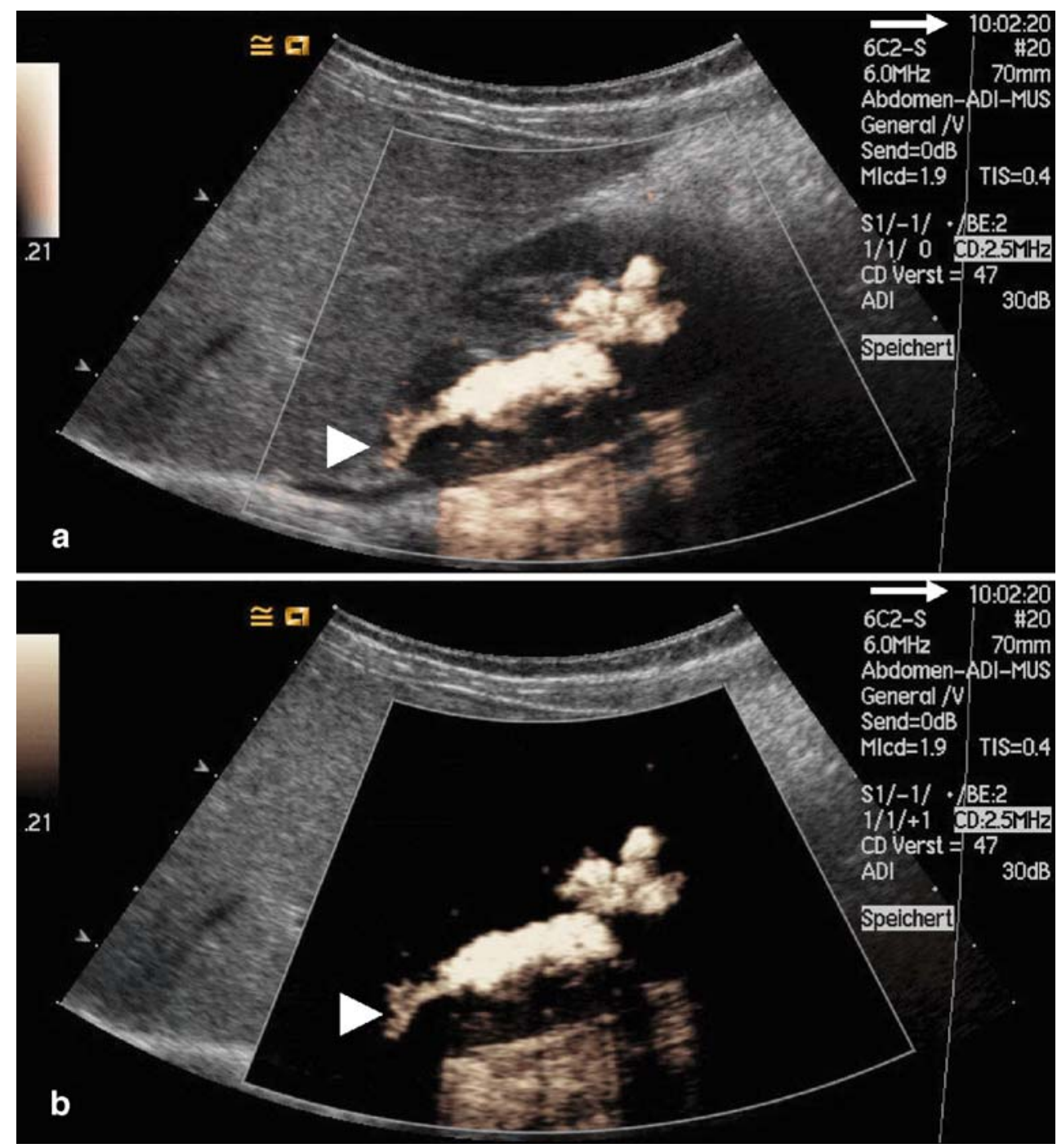

scan in the supine position may suffice if adequate visualization of the renal pelves on both sides is possible, even though scanning the kidneys with the child in the prone position definitely allows better demonstration of the pelvicalyceal system. It should always be kept in mind that not performing a precontrast scan reduces the duration of the whole examination. This should be the case whenever harmonic or other dedicated contrast imaging is used.

\section{Intravesical administration of normal saline and US contrast agent}

The UCA and normal saline are administered via a transurethral catheter, but suprapubic puncture is also possible. The administration of UCA is carried out under US monitoring. The aim should be to have homogeneous strong contrast agent density of the bladder contents. Early studies advocated filling the bladder to the maximum prior to injecting the UCA $[13,28]$. This makes the calculation of UCA volume to be administered easier, but has the disadvantage that low-pressure reflux may be obscured. Moreover, in neonates and infants due to repeated voiding at small bladder filling volume, there is insufficient time to administer UCA and scan the urinary tract. Increasingly, a more fractional approach to administering UCA is being practised $[21,40]$. It is important to note that emptying the bladder for the sake of UCA administration is not mandatory as explained above. It is helpful to inject UCA into a bladder that is not completely empty in order to have better visualization of the microbubbles and avoid a strong dorsal acoustic shadow. Using a three-way valve it is easy to alternately administer normal saline and UCA adjusting the volume of UCA to the bladder filling volume and in the end reaching the recommended dose. The Levovist suspension should be administered slowly for two reasons: first, to minimize the destruction of the microbubbles and 
second, to reduce settling of the suspension at the bottom of the bladder that would create an acoustic shadow that temporarily obscures the retrovesical region [28]. In such a case, turning the child several times from side to side hastens the homogeneous distribution of the UCA in the bladder. The administration of normal saline is continued until the child has the urge to micturate or there is the first slight sign of back pressure to the infusion or injection.

\section{Postcontrast scan of the bladder, ureters and kidneys}

The US scan after contrast agent administration is basically carried out in the same manner as that before contrast agent administration. There are minor procedural variations depending on the type of US modality utilized. The diagnosis of reflux is made when echogenic microbubbles appear in a ureter or renal pelvis. When a ureter is distinctly visible behind the bladder, the microbubbles render the echo-free lumen echogenic. When the ureter is not clearly visible as a round echo-free structure, VUR can be demonstrated if microbubbles are seen entering the vesicoureteric junction or if just behind this junction, one can depict a round echogenic spot in transverse section, which can be differentiated from its surroundings by its strong echogenicity and possibly constant movement of microbubbles [28]. When the reflux reaches the kidney the echogenic microbubbles can be detected in the pelvicalyceal system. In a recent study by Kopac et al. [23] the need for UCA to demonstrate reflux was again emphasized. They compared indirect non-contrast VUS with contrastenhanced VUS in 47 children with 93 PUUs. In the former there was no catheterization and bladder filling and any increase in renal pelvic and proximal ureteric width during voiding was considered as a sign of VUR. Compared to VUS with UCA this was found to have low diagnostic accuracy of only $59 \%$, which is not sufficiently reliable for routine use.

\section{Postcontrast scan of the bladder, ureters and kidneys during and after voiding}

The US examination is continued during and after voiding in a similar manner as above, always scanning the right and left kidneys alternately, and also the bladder if the patient's position allows. If cyclic filling is not planned, the catheter can be removed prior to micturition. Most children can void around a thin catheter on the examination table while lying supine, prone or on the side [28]. Other alternatives to consider are to have the child sit on a potty and to scan the kidneys from the back. A "music potty" that signals voiding with music is helpful. In older boys the option to micturate into a urine bottle while standing and being scanned from the back may be offered. At the end the bladder is checked for residual urine.
Postcontrast scan of the urethra during voiding (urethrosonography)

The main focus in VUS has been on detection of reflux. In the past, transperineal imaging of the urethra was not given as much consideration during VUS. The lack of urethral imaging in VUS was regarded as a drawback compared to VCUG $[46,47]$. In recent years there has been an increased interest in the inclusion of urethral imaging. To date, six studies are available that deal exclusively or partly with contrast-enhanced voiding US of the urethra [16, 19, 4851]. In these studies the urethra was examined in a total of 847 children comprising 647 (76\%) boys and 200 (24\%) girls (age range 1 day to 15 years). Levovist in combination with fundamental imaging was used in all children. In half of the studies, both boys and girls were included and in the remainder only boys $[19,48,49]$. An unbiased comparison of all cases with VCUG was carried out in only two studies $[48,49]$. In three studies just those children with pathological findings of the urethra by voiding urethrosonography underwent VCUG [19, 50, 51]. In the remaining one study the selection for comparison was not clearly presented [16]. The diagnosis of posterior urethral valve was made in 22 boys $(3.3 \%)$ using transperineal contrast-enhanced voiding urethrosonography (Fig. 5). This was also found to be suitable for assessment of the urethra after valve resection $[48,49]$. Other diagnoses in boys were anterior urethral valve and urethral stenosis [48]. All diagnoses were confirmed by VCUG. However, the urethral pathologies detected were not only too few, but also the range of pathologies encountered was small [47]. Other urethral pathologies such as paraurethral cysts, diverticula, double urethra, urethral fistula, and complex anorectal and cloacal malformations are still missing in these series. Urethrovaginal reflux was evaluated in two studies and was found to be present in 67 of 165 girls $(40.6 \%)$ [19, 49].

The most important study comparing contrast-enhanced voiding urethrosonography and VCUG is that by Berrocal et al. [49]. In this prospective study a total of 146 children ( 87 boys, 59 girls) with a mean age of 3.3 years ( 8 days to 14 years) were recruited. In the voiding part of VUS, primarily transperineal US of the urethra was performed. During voiding the catheter was removed. The diameters of the anterior and posterior parts of the urethra were measured at maximum dilatation during voiding. In boys the normal value was found to be $6.1 \pm 0.8 \mathrm{~mm} \mathrm{(2.8-}$ $7.1 \mathrm{~mm})$ and $6.3 \pm 0.67 \mathrm{~mm}(3.7-7.2 \mathrm{~mm})$, respectively. In girls the urethral diameter was $4.2 \pm 1.0 \mathrm{~mm}(2.5-7.8 \mathrm{~mm})$. This was the basis for evaluation of pathological changes in the urethra. In all children the US study was followed by VCUG. There were three boys with posterior urethral valves detected on US with dilated posterior urethra, reduced diameter of the anterior urethra and delay in 
Fig. 5 Transperineal voiding urethrosonography (a) as part of VUS in comparison with (b) VCUG. To facilitate the comparison the US image (a) is presented upside down. Note in the transperineal US (a) the microbubbles in the bladder $(B)$ and in the massively dilated posterior urethra $(p U)$. The anterior urethra $(a U)$ is depicted as very thin in the presence of a posterior urethral valve (arrow). The finding was confirmed on VCUG (b) (courtesy of Dr. M. Bosio, Milan, Italy)
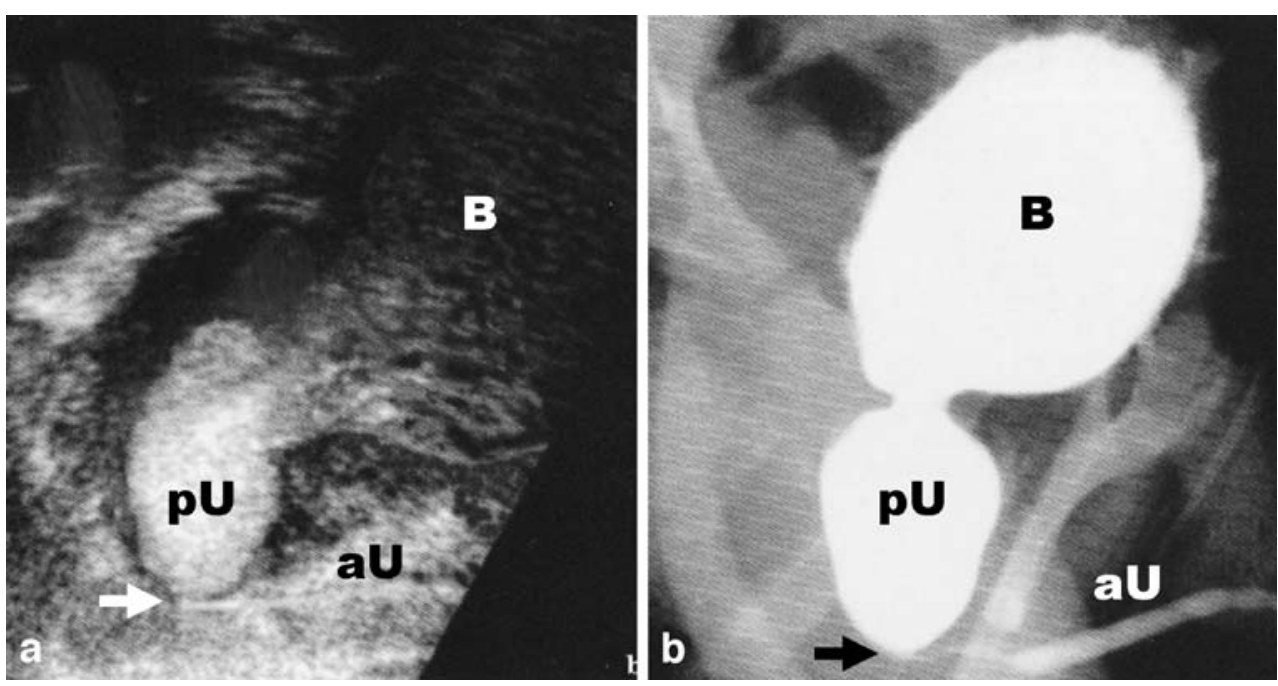

UCA flow at the valve. Furthermore, urethral stenosis was diagnosed in two boys. Seven children were evaluated after resection of the posterior urethra valves and were found to have a normal posterior urethral diameter in the presence of dilatation of the anterior part without any flow delay. In all children these findings were confirmed on VCUG. All girls and 75 boys showed a normal urethra at both transperineal US and VCUG. Thus sensitivity and specificity were each $100 \%$. The approximate mean duration for VUS including transperineal voiding US was $30 \mathrm{~min}$. In conclusion, transperineal contrast-enhanced VUS of the urethra has not only been shown to be possible but also to be a potential adjunct to VUS in routine examinations.

\section{Cyclical filling of the bladder}

VUR is an intermittent phenomenon. Differences in presence and degree of reflux on repeated examinations have been reported in both VCUG and RNC [52, 53]. In general, cyclical filling results in an increase in reflux detection rate. Repeated filling of the bladder may be necessary when there is marked discrepancy in kidney size and/or intermittent dilatation of the ureters on US and a negative result during the initial examination. VUS has a specific advantage in this regard as it is not associated with repeated exposure to radiation. Three studies have evaluated the value of cyclical filling in VUS [54-56]. In two of the studies, two cycles of bladder filling including Levovist administration were carried out with scanning in fundamental mode [54, 55]. One study was performed in 27 patients with transplanted kidneys [54]. This group comprised eight children (age range 12.2-18.3 years) and 19 adults (age range 19.2-54.8 years). The reflux detection rate in the first and second cycles was 17 and 16 of 27 , respectively, and thus not very different, but there were significantly higher reflux grades in the second cycle. So the conclusion was that cyclical filling in VUS with fundamental imaging did not result in increased reflux detection rate. In another study with a greater number and wider spectrum of patients, exclusively children, the opposite was found. Novljan et al. [55] evaluated cyclical filling in 49 children (age range 1.4-15.8 years, mean 4.1 years) with 98 PUUs. A total of 35 PUUs with reflux were detected when taking both cycles into account. In the first cycle 28 of 35 and in the second 33 of 35 of the PUUs were positive. In the first cycle only just 2 of 35 refluxes (both grade II) were detected. The VUR diagnosed in the second cycle alone comprised six PUUs with grade II and one PUU with grade III reflux. Not only were $25 \%$ more refluxing units detected in the second cycle but also $50 \%$ more grade III refluxes.

When the bladder is completely emptied one can often still find residual echogenic UCA lining the bladder mucosa which together with the hypoechoic wall resembles the mouth part of a "smile sign" (Fig. 6). This so-called "smile sign" indicates that there is still enough UCA in the bladder to facilitate repeat filling with normal saline alone and allow another cycle of VUS. Papadopoulou et al. [56] found that in 112 of 117 children (96\%) undergoing cyclical harmonic VUS the residual UCA in the bladder was sufficient to allow a second filling with normal saline only. In the 112 children (224 PUUs) they compared the results of the first cycle with Levovist and normal saline administration with those of a second cycle with administration of only normal saline. VUR was detected in 57 PUUs in the first cycle, and of the remaining 167 nonrefluxing PUUs, 12 showed VUR in the second cycle (one grade I, nine grade II, two grade III). Only one PUU reflux (grade 


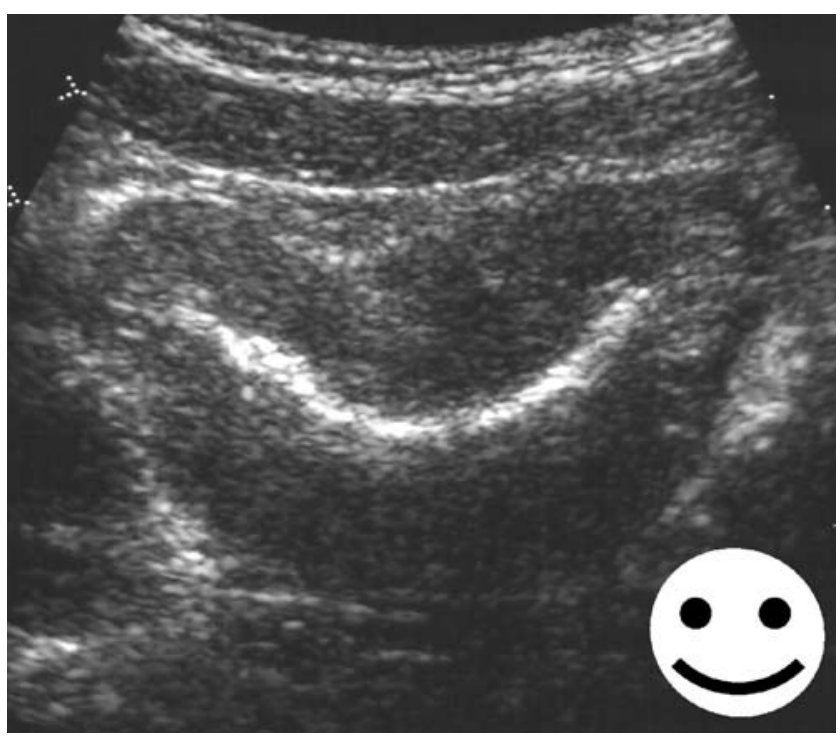

Fig. 6 At the end of VUS and bladder emptying there can still be a "layer" of UCA lining the mucosa of the empty bladder. The configuration of the bladder in transverse section in combination with the remaining echogenic UCA resembles the mouth part of a "smile sign". This sign indicates that there is still sufficient UCA in the bladder and if considered necessary a second filling with normal saline only would suffice to carry out cyclical VUS

II) was diagnosed in the first cycle but missed in the second cycle. Considering all refluxing units as true positives, the sensitivities of the first and second cycles were $87 \%$ and $98 \%$ and the negative predictive values $84 \%$ and $98 \%$, respectively. Thus a second cycle of harmonic VUS with no addition of UCA reveals significantly more PUUs with VUR at almost no additional cost for the examination. From the current standpoint of available data, cyclical filling in VUS, particularly when using harmonic imaging, seems to be a very promising adjunct.

\section{Grading of reflux}

VUR grading is necessary because the severity of reflux correlates with prognosis and consequently serves as a basis for therapeutic decision making. It is also essential to have a uniform grading system for comparison between different research studies. The reflux gradings are based on the degree of pelvicalyceal and ureteral dilatation. Intrarenal reflux is not taken into consideration for grading purposes. It is to be noted that it is possible to depict intrarenal reflux in VUS, particularly when using harmonic imaging and other high-MI modalities $[13,57]$. No systematic evaluation of intrarenal reflux in VUS is available. The first grading system for VUS was proposed in 1985 by Beyer et al. [58]. Here grading was for VUS with administration of normal saline only into the bladder. This sonographic grading has five levels based on the extent of dilatation of the renal pelvis, calyces and ureters. Taking this grading system and in conformity with the international reflux grading for VCUG, a five-level grading system was also adapted for contrast-enhanced VUS [59, 60]. The diagnosis of reflux is based on the presence of microbubbles and the severity of the reflux determined by taking primarily the pelvicalyceal and secondarily the ureteric dilatation into account (Table 1). This grading system for VUS has gained widespread acceptance. With the severity of reflux being a continuum, no sonographic measurements have been proposed for differentiating between the grades. Unavoidably, as in VCUG, there is a certain element of subjectivity in the grading system and also some reflux episodes do not fall precisely within one of the five grades. The attempt to differentiate between reflux in dilated and nondilated systems has not become widely used, probably due to lack of immediate consequence of such an addition [60].

\section{Duration of VUS examination}

In four comparative studies, in which VUS and VCUG were carried out successively, the duration of each procedural step was recorded [12, 28, 31, 42]. In two of the studies VUS was carried out using fundamental mode $[12,28]$. The other two studies incorporated colour Doppler US [31, 42]. In these studies the durations of VUS, including catheterization, were in the range 26-34 min with the precontrast US taking up almost one-third of the time. The durations of VCUG, adding the time for catheterization, were in the range $13-20 \mathrm{~min}$. In one other study VUS and VCUG were carried out simultaneously and here the duration of one such examination including catheterization was 9 min [61]. Novljan et al. [55] performed VUS with cyclical filling (two fillings) and the mean duration of the VUS was 24 min (range 15-30 min). Overall, these studies show that the duration of VUS shows a wide range and is longer than that of VCUG. As mentioned above the precontrast US may not be necessary when using harmonic or other dedicated contrast imaging modalities, resulting in a reduction of the duration of VUS by almost one-third. This would put the duration of VUS equivalent to that of VCUG.

Table 1 Reflux grading in contrast-enhanced VUS

\begin{tabular}{ll}
\hline Grade & Definition \\
\hline I & Microbubbles only in the ureter \\
II & Microbubbles in the renal pelvis; no significant renal pelvic \\
& dilatation
\end{tabular}



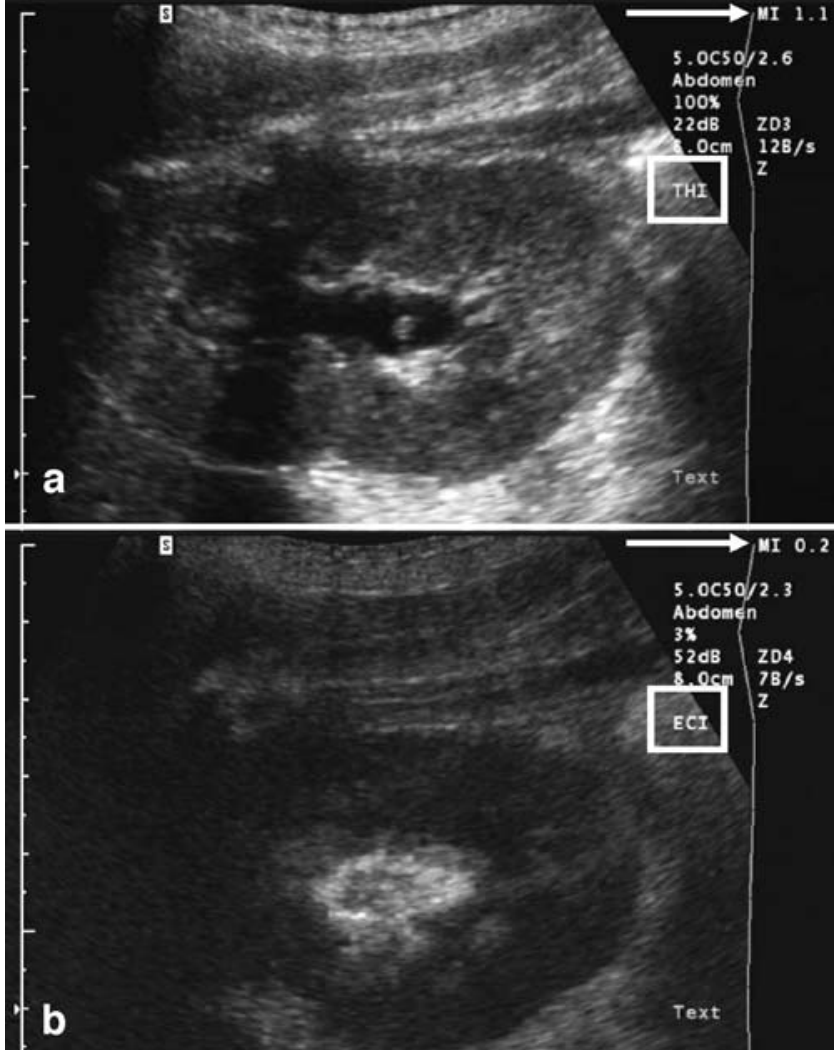

Fig. 7 VUS using the second-generation UCA, SonoVue. The scan before contrast agent administration (a) uses the tissue harmonic imaging $(T H I)$ modality and the scan after contrast agent administration (b) uses echo contrast imaging (ECI). In the former the MI (arrow) is high (1.1) while in the latter a low-MI (0.2) technique is applied. In THI the kidney and particularly the renal pelvis are very well depicted. The refluxing microbubbles of the second-generation UCA are displayed most conspicuously using the low-MI imaging modality

\section{VUS with a second-generation US contrast agent (SonoVue)}

In 2001 a second-generation UCA-SonoVue (Bracco, Milan, Italy) - was approved in the European Union for IV use in adults. SonoVue is now widely used, the main application being for the evaluation parenchymal abdominal lesions [62]. Even though it has not been approved yet for use in children one in-vitro and four clinical studies point out potential advantages compared to the use of the first-generation UCA, Levovist [63-67]. SonoVue is composed of a stabilized aqueous suspension of sulphur hexafluoride $\left(\mathrm{SF}_{6}\right)$ microbubbles with a phospholipid shell and is available as a package comprising one vial with granules and a 5-ml syringe prefilled with normal saline [62]. In an in-vitro comparative study with Levovist (5\% volume) the contrast duration with SonoVue was seven times longer at a dose that was $80 \%$ lower [63]. It was found that UCA at $0.25-1.0 \%$ of the filling volume would provide adequate contrast. Moreover, the contrast duration of a freshly prepared suspension of SonoVue was stable over $6 \mathrm{~h}$, whereas Levovist showed a significant reduction after $30 \mathrm{~min}$.

The first clinical studies [64-67], in total comprising 210 children, have shown that low-MI imaging is the most favourable US modality for VUS when using SonoVue [67] (Fig. 7). Although it is possible to use all other modalities with this UCA, the highest contrast difference between tissue and microbubbles seems to be achieved with low-MI imaging, in which the tissue is suppressed and the microbubbles become more conspicuous. During intravesical administration of SonoVue there is a minor difference compared with Levovist. With the patient in the supine position at the beginning of injection the UCA accumulates at the roof of the bladder and thus does not reach the vesicoureteric junctions [65]. Only after starting normal saline infusion can a homogeneous distribution of the microbubbles be observed. Not much acoustic shadowing of the retrovesical space is encountered. The intravesical dosages that have been successfully used in clinical studies are $1 \%$ of bladder filling [65] and $1 \mathrm{ml}$ per bladder filling [67]. This implies that with a 5-ml suspension from one vial that is stable over $6 \mathrm{~h}$ there is the potential for performing several studies from the one vial. If one vial can be used for more than one patient, the cost of the UCA, a major obstacle to the widespread use of VUS, could be reduced. It is important to note that in some countries there may be restrictions regarding the use of one vial for more than one patient.

\section{Contraindications and adverse events from intravesical administration of US contrast agents}

There are no specific contraindications as such to the intravesical administration of Levovist. There are five clinical studies including a total of 626 patients with an age range of 2 days to 20 years, in which systematic monitoring for possible adverse events related to intravesical administration of Levovist were carried out $[12,20$, $28,41,42]$. The evaluation incorporated all or some of the following: various levels of vital sign monitoring [12, 28], assessment for possible signs and symptoms during and after the procedure, observation for up to $12 \mathrm{~h}$ as inpatient [42], and request to the patient and parents to report any symptoms in the next $24 \mathrm{~h}$ and an active 24-h follow-up by phone. This evaluation was carried out within the context of comparative studies, in which VCUG was performed following VUS. All patients were catheterized for the examinations. There were 15 patients with transient visible haematuria during or at completion of voiding at either VUS or VCUG and one patient each with transient mild 
abdominal and urethral pain. For these observed adverse events the catheterization was primarily to blame rather than the UCA [68]. No substance-specific adverse events definitely related to the intravesical administration of Levovist were observed.

The osmolality of Levovist in solution is about five times higher than that of blood [37]. This osmolality corresponds to that of previously used ionic radiographic contrast agents, which were likewise instilled into the bladder. No adverse events attributable to the osmolality were observed with these agents. Apart from this, the amount of UCA administered is small and in the bladder there is usually urine and/or normal saline, resulting in dilution of the hyperosmolar solution. Hyperosmolality of the UCA can, therefore, be ignored in VUS, particularly as it lies approximately in the physiological range of osmolality of urine. No animal study has been published on the potential effect of insonation of the urothelium in the presence of microbubbles, particularly at high MI settings. Potential alterations of the urothelium cannot be excluded with absolute certainty. Over the last 10 years, predominantly in Europe, it is roughly estimated that some 20,000 VUS examinations have been conducted in children (publications, reports and personal communications). There have been no reports of clinical adverse effects related to the intravesical administration of Levovist.

Experience with the new UCA, SonoVue, for VUS is still very limited. Contraindications for the IV administration are known hypersensitivity to sulphur hexafluoride and certain cardiopulmonary disorders [29]. Recently, a largescale retrospective analysis showed that IV SonoVue has a good safety profile in abdominal applications [69]. In the small number of VUS studies with a total of 210 children no adverse events directly related to the UCA have been reported [64-67]. There are, as yet, no detailed studies regarding any possible interaction with urine and the urothelium.

\section{Conclusion}

It is important to realize that a breakthrough to implement US for reflux diagnosis, which had began in the late 1970s, only came with the availability of a stable UCA. Advances in US technology resulting in marked improvement in the depiction of microbubbles have facilitated many procedural aspects in addition to the diagnostic accuracy of the modality. Furthermore, the first results of VUS with the use of a more stable second-generation UCA point to further procedural and diagnostic progress. Like VCUG and direct RNC, it is still necessary to catheterize the bladder or perform suprapubic puncture for VUS. This is a major drawback of all direct reflux examinations and one that makes them invasive. Attempts to generate bubbles exogenously using high-intensity focused US would solve this problem, but have not yet moved beyond the stage of animal studies [70].

\section{References}

1. Darge K (2002) Diagnosis of vesicoureteral reflux with ultrasound. Pediatr Nephrol 17:52-60

2. Kaneko K, Kuwatsuru R, Fukuda Y et al (1994) Contrast sonography for detection of vesicoureteral reflux. Lancet 344:687

3. von Rohden L, Bosse U, Wiemann D (1995) Refluxsonographie bei Kindern mit einem Ultraschallkontrastmittel im Vergleich zur Röntgenmiktionszystourethro-graphie. Paediat Prax 49:49-58

4. McEwing RL, Anderson NG, Hellewell S et al (2002) Comparison of echo-enhanced ultrasound with fluoroscopic MCU for the detection of vesicoureteral reflux in neonates. Pediatr Radiol 32:853-858

5. Mentzel HJ, Vogt S, Patzer L et al (1999) Contrast-enhanced sonography of vesicoureterorenal reflux in children: preliminary results. AJR 173:737-740

6. Vassiou K, Vlychou M, Moisidou R et al (2004) Contrast-enhanced sonographic detection of vesicoureteral reflux in children: Comparison with voiding cystourethrography. Rofo 176:1453-1457

7. Radmayr C, Klauser A, Pallwein L et al (2002) Contrast enhanced reflux sonography in children: a comparison to standard radiological imaging. J Urol 167:1428-1430

8. Elias P, Rejtar P, Sylva S et al (1999) Preliminary experience with contrast-enhanced ultrasound cystography in the diagnosis of vesicoureteral reflux. Ces Radiol 53(Suppl 1):4-8

9. Escape I, Martinez J, Bastart F et al (2001) Usefulness of echocystography in the study of vesicoureteral reflux. J Ultrasound Med 20:145-149

10. Farina R, Arena C, Pennisi F et al (1999) Retrograde cystography US: a new ultrasound technique for the diagnosis and staging of vesicoureteral reflux. Radiol Med 97:360-364

11. Ascenti G, Chimenz R, Zimbaro G et al (2000) Potential role of colour-Doppler cystosonography with echocontrast in the screening and follow-up of vesicoureteral reflux. Acta Paediatr 89:1336-1339

12. Berrocal T, Gaya F, Arjonilla A et al (2001) Vesicoureteral reflux: diagnosis and grading with echo-enhanced cystosonography versus voiding cystourethrography. Radiology 221:359-365

13. Bosio M (1998) Cystosonography with echocontrast: a new imaging modality to detect vesicoureteric reflux in children. Pediatr Radiol 28:250-255

14. Piaggio G, Degl' Innocenti ML, Toma P et al (2003) Cystosonography and voiding cystourethrography in the diagnosis of vesicoureteral reflux. Pediatr Nephrol 18:18-22

15. Riccabona M, Mache CJ, Lindbichler F (2003) Echo-enhanced color Doppler cystosonography of vesicoureteral reflux in children: improvement by stimulated acoustic emission. Acta Radiol 44:18-23

16. Xhepa R, Bosio M, Manzoni G (2004) Voiding cystourethrosonography for the diagnosis of vesicoureteral reflux in a developing country. Pediatr Nephrol 19:638-643

17. Darge K, Zieger B, Rohrschneider W et al (2001) Contrastenhanced harmonic imaging for the diagnosis of vesicoureteral reflux. AJR 177:1411-1415

18. Kopitzko A, Cornely D, Reither K et al (2004) Low contrast dose voiding urosonography in children with phase inversion imaging. Eur Radiol 14:2290-2296

19. Maté A, Bargiela A, Mosteiro S et al (2003) Contrast ultrasound of the urethra in children. Eur Radiol 13:1534-1537 
20. Mentzel HJ, Vogt S, Joan U et al (2002) Voiding urosonography with ultrasonography contrast medium in children. Pediatr Nephrol 17:272-276

21. Nakamura M, Shinozaki T, Taniguchi N et al (2003) Simultaneous voiding cystourethrography and voiding urosonography reveals utility of sonographic diagnosis of vesicoureteral reflux in children. Acta Paediatr 92:1422-1426

22. Uhl M, Kromeier J, Zimmerhackl LB et al (2003) Simultaneous voiding cystourethrography and voiding urosonography. Acta Radiol 44:265-268

23. Kopac M, Kenig A, Kljucevsek D et al (2005) Indirect voiding urosonography for detecting vesicoureteral reflux in children. Pediatr Nephrol 20:1285-1287

24. Darge K, Troeger J (2000) Sonographic examination of vesicoureterorenal reflux: one method many names! Proceedings of the Second European Meeting on Sonographic Diagnosis of Vesicoureteral Reflux, Heidelberg, Germany

25. Acronym Finder (2007) Mountain Data Systems, Estes Park. http://www.acronymfinder.com. Cited 8 June 2007

26. Darge K (2007) Voiding urosonography with US contrast agent for the diagnosis of vesicoureteric reflux in children. II. Comparison with radiologic examinations. Pediatr Radiol DOI 10.1007/s00247-0070528-8

27. Fritzsch T, Schlief R (1995) Levovist. SHU-508-A. Drugs Fut 20:1224-1227

28. Darge K, Troeger J, Duetting T et al (1999) Reflux in young patients: comparison of voiding US of the bladder and retrovesical space with echo enhancement versus voiding cystourethrography for diagnosis. Radiology 210:201-207

29. Albrecht T, Blomley M, Bolondi L et al (2004) Guidelines for the use of contrast agents in ultrasound. January 2004. Ultraschall Med 25:249-256

30. Kmetec A, Bren AF, Kandus A et al (2001) Contrast-enhanced ultrasound voiding cystography as a screening examination for vesicoureteral reflux in the follow-up of renal transplant recipients: a new approach. Nephrol Dial Transplant 16:120-123

31. Farina R, Arena C, Pennisi F et al (2000) Vesicoureteral reflux: diagnosis and staging with voiding color doppler US. Preliminary experience. Eur J Radiol 35:49-53

32. Koff SA (1983) Estimating bladder capacity in children. Urology $21: 248$

33. Darge K, Moeller RT, Trusen A et al (2004) Diagnosis of vesicoureteric reflux with low-dose contrast-enhanced harmonic ultrasound imaging. Pediatr Radiol 35:73-78

34. Radiological Society of North America (2005) RSNA 2005, 27 November to 2 December, Chicago. Session code: RC813B Sonographic evaluation of vesicoureteric reflux. Radiological Society of North America, Oak Brook

35. Darge K, Rössling G (2000) In-vitro simulation of Levovist-enhanced VUS: influence of various physical parameters on the microbubbles. Proceedings of the Second European Meeting on Sonographic Diagnosis of Vesicoureteral Reflux, Heidelberg, Germany

36. Darge K, Bruchelt W, Roessling G et al (2003) Interaction of normal saline solution with ultrasound contrast medium: significant implication for sonographic diagnosis of vesicoureteral reflux. Eur Radiol 13:213-218

37. Nanda NC, Schlief R, Goldberg B (1997) Advances in echo imaging using contrast enhancement. Kluwer, Dordrecht, The Netherlands, pp 85-113, 71-96

38. Rössling G (2000) Physico-chemical properties of Levovist. Proceedings of the Second European Meeting on Sonographic Diagnosis of Vesicoureteral Reflux, Heidelberg, Germany

39. Darge K, Roessling G, Troeger J (2003) Do microbubbles ascend passively in the ureter? Pediatr Radiol 33:S49

40. Darge K, Riedmiller H (2004) Current status of vesicoureteral reflux diagnosis. World J Urol 22:88-95
41. Valentini AL, Salvaggio E, Manzoni C et al (2001) Contrastenhanced gray-scale and color Doppler voiding urosonography versus voiding cystourethrography in the diagnosis and grading of vesicoureteral reflux. J Clin Ultrasound 29:65-71

42. Galia M, Midiri M, Pennisi F et al (2004) Vesicoureteral reflux in young patients: comparison of voiding color Doppler US with echo enhancement versus voiding cystourethrography for diagnosis or exclusion. Abdom Imaging 29:303-308

43. Tranquart F, Grenier N, Eder V et al (1999) Clinical use of ultrasound tissue harmonic imaging. Ultrasound Med Biol 25: 889-894

44. Bartram U, Darge K (2005) Harmonic versus conventional ultrasound imaging of the urinary tract in children. Pediatr Radiol 35:655-660

45. Youk JH, Kim CS, Lee JM (2003) Contrast enhanced agent detection imaging - value in the characterization of focal hepatic lesions. J Ultrasound Med 22:897-910

46. Riccabona M (2002) Cystography in infants and children: a critical appraisal of the many forms with special regard to voiding cystourethrography. Eur Radiol 12:2910-2918

47. Riccabona M (2003) Contrast ultrasound of the urethra in children. Eur Radiol 13:1494-1495

48. Berrocal T, Rivas S, Jaureguizar E et al (2004) Contrast-enhanced sonourethrography in the assessment of the urethra. Cir Pediatr $17: 58-60$

49. Berrocal T, Gaya F, Arjonilla A (2005) Vesicoureteral reflux: can the urethra be adequately assessed by using contrast-enhanced voiding US of the bladder? Radiology 234:235-241

50. Bosio M, Manzoni GA (2002) Detection of posterior urethral valves with voiding cystourethrosonography with echo contrast. J Urol 168:1711-1715

51. Bosio M (2002) Role of ultrasound in the imaging of posterior urethral valves. Rays 27:135-139

52. Gelfand MJ, Koch BL, Elgazzar AH et al (1999) Cyclic cystography: diagnostic yield in selected pediatric populations. Radiology 213:118-120

53. Fettich JJ, Kenda RB (1992) Cyclic direct radionuclide voiding cystography: increasing reliability in detecting vesicoureteral reflux in children. Pediatr Radiol 22:337-338

54. Kenda RB, Kenig A, Novljan G et al (2001) Cyclic voiding urosonography for detecting vesicoureteric reflux in renal transplant recipients. Nephrol Dial Transplant 16:2229-2231

55. Novljan G, Kenig A, Rus R et al (2003) Cyclic voiding urosonography in detecting vesicoureteral reflux in children. Pediatr Nephrol 18:992-995

56. Papadopoulou F, Tsampoulas C, Siomou E (2006) Cyclic contrastenhanced harmonic voiding urosonography for the evaluation of reflux. Can we keep the cost of the examination low? Eur Radiol $16: 2521-2526$

57. Darge K, Trusen A, Gordjani N et al (2003) Intrarenal reflux: diagnosis with contrast-enhanced harmonic US. Pediatr Radiol 33:729-731

58. Beyer HJ, Hofmann V, Brettschneider D (1985) The micturition sonourogram: a new possibility of determining vesicoureteral reflux in childhood. Ultraschall Med 6:182-188

59. Lebowitz RL, Olbing H, Parkkulainen KV et al (1985) International system of radiographic grading of vesicoureteric reflux. Pediatr Radiol 15:105-109

60. Darge K, Troeger J (2002) Vesicoureteral reflux grading in contrast-enhanced voiding urosonography. Eur $\mathrm{J}$ Radiol 43:122-128

61. Nakamura M, Wang Y, Shigeta K et al (2002) Simultaneous voiding cystourethrography and voiding urosonography: an in vitro and in vivo study. Clin Radiol 57:846-849

62. Greis C (2004) Technology overview: SonoVue (Bracco, Milan). Eur Radiol Suppl 8:11-15 
63. Robrecht J, Darge K (2007) In-vitro comparison of a 1st and a 2nd generation US contrast agent for reflux diagnosis. RoFo DOI $10.1055 / \mathrm{s}-2007-963312$

64. Anthopoulou A, Fotopoulos A, Tzovara J et al (2005) Comparison of voiding urosonography harmonic imaging using 2 nd generation contrast agent with direct radionuclide cystography for the diagnosis of reflux. Eur Radiol S15:336

65. Darge K, Beer M, Gordjani N et al (2004) Contrast-enhanced voiding urosonography with the use of a 2 nd generation US contrast medium: preliminary results. Pediatr Radiol 34:S97

66. Papadopoulou F, Katzioti F, Arkoumani E et al (2005) Voiding urosonography harmonic imaging with 2 nd generation contrast agent for the diagnosis of reflux. Pediatr Radiol 35:S130
67. Ascenti G, Zimbaro G, Mazziotti S et al (2004) Harmonic US imaging of vesicoureteric reflux in children: usefulness of a second generation US contrast agent. Pediatr Radiol 34:481-487

68. Zerin MJ, Shulkin BL (1992) Postprocedural symptoms in children who undergo imaging studies of the urinary tract: is it the contrast material or the catheter? Radiology 182:727-730

69. Piscaglia F, Bolondi L (2006) The safety of SonoVue in abdominal applications: retrospective analysis of 23188 investigations. Ultrasound Med Biol 32:1369-1375

70. Fowlkes JB, Carson PL, Chiang EH et al (1991) Acoustic generation of bubbles in excised canine urinary bladder. J Acoust Soc Am 89:2740-2744 